\title{
Quasi-equational bases for graphs of semigroups, monoids and groups
}

\author{
Michał M. Stronkowski
}

Received: 13 April 2010 / Accepted: 14 September 2010 / Published online: 19 October 2010

(C) The Author(s) 2010. This article is published with open access at Springerlink.com

\begin{abstract}
The graph of an algebra $A$ is the relational structure $G(A)$ in which the relations are the graphs of the basic operations of $\mathbf{A}$. Let denote by $\mathbf{G}(\mathscr{C})$ the class of all graphs of algebras from a class $\mathscr{C}$. We prove that if $\mathscr{C}$ is a class of semigroups possessing a nontrivial member with a neutral element, then $G(\mathscr{C})$ does not have finite quasi-equational basis. We deduce that, for a class $\mathscr{C}$ of monoids or groups with a nontrivial member, $\mathrm{G}(\mathscr{C})$ also does not have finite quasi-equational basis.
\end{abstract}

Keywords Graphs of semigroups · Finite axiomatizability · Finite quasi-equational bases - Quasivarieties of relational structures

\section{Introduction}

The graph of an algebra $\mathbf{A}=(A, O)$ is the relational structure

$$
\mathbf{G}(\mathbf{A})=\left(A,\left\{R_{o} \mid o \in O\right\}\right),
$$

where each $R_{o}$ is the graph of an operation $o$. This means that if $o$ is an $n$-ary operation, then $R_{o}$ is the $(n+1)$-ary relation consisting of those tuples $\left(a_{1}, \ldots, a_{n+1}\right)$ which satisfy $o\left(a_{0}, \ldots, a_{n-1}\right)=a_{n}$. Most of the time we will work with groupoids

Communicated by Mikhail Volkov.

The author was supported by the Eduard Čech Center Grant LC505 and by the Statutory Grant of Warsaw University of Technology 504G11200112000.

M.M. Stronkowski (凶)

Faculty of Mathematics and Information Science, Warsaw University of Technology, Warsaw, Poland e-mail: m.stronkowski@mini.pw.edu.pl

M.M. Stronkowski

Eduard Čech Center, Charles University, Prague, Czech Republic 
(semigroups in fact) and, without any risk of ambiguity, we will omit the subscript $o$. For a class $\mathscr{C}$ of algebras by $\mathrm{G}(\mathscr{C})$ we denote the class of all graphs of algebras from $\mathscr{C}$.

We are mainly interested in quasi-equational theory of graphs of algebras. Following $[8,14]$ we call a sentence quasi-identity if it is of the form

$$
(\forall \bar{x})\left[\varphi_{1}(\bar{x}) \wedge \cdots \wedge \varphi_{n}(\bar{x}) \rightarrow \varphi(\bar{x})\right],
$$

where $n \in \mathbb{N}$ and $\varphi_{1}, \ldots, \varphi_{n}, \varphi$ are atomic formulas. We allow $n$ to be zero, and in such case we call the sentence an identity. By a quasi-equational theory of a class $\mathscr{K}$ we mean the set of quasi-identities true in $\mathscr{K}$. A quasivariety is a class defined by quasi-identities or, equivalently, a class which is closed under taking substructures, direct products and ultraproducts (we tacitly assume that all considered classes are closed under taking isomorphic images). The smallest quasivariety containing a class $\mathscr{K}$, i.e. the class defined by the quasi-equational theory of $\mathscr{K}$, is denoted by $\mathrm{Q}(\mathscr{K})$. Any defining set of quasi-identities for $\mathrm{Q}(\mathscr{K})$ is called a quasi-equational basis of $\mathscr{K}$. By compactness theorem, $\mathrm{Q}(\mathscr{K})$ is finitely axiomatizable iff $\mathscr{K}$ has a finite quasi-equational basis.

In this paper we wish to attract the reader's attention to the following, elementary but curious, fact.

Theorem 1 Let $\mathscr{C}$ be a class of semigroups possessing a nontrivial member with a neutral element. Then $\mathrm{G}(\mathscr{C})$ does not have a finite quasi-equational basis.

Note that the existence of a nontrivial semigroup in $\mathscr{C}$ would not be a sufficient assumption in the above theorem. For example, if $\mathscr{Z}$ is the class of semigroups satisfying $\left(\forall x, y, x^{\prime}, y^{\prime}\right)\left[x y \approx x^{\prime} y^{\prime}\right]$, then $\mathrm{QG}(\mathscr{Z})$ is axiomatized by

$$
\begin{aligned}
& \left(\forall x, y, z, z^{\prime}\right)\left[R(x, y, z) \wedge R\left(x, y, z^{\prime}\right) \rightarrow z \approx z^{\prime}\right], \\
& \left(\forall x, y, x^{\prime}, y^{\prime}, z\right)\left[R(x, y, z) \rightarrow R\left(x^{\prime}, y^{\prime}, z\right)\right] .
\end{aligned}
$$

In the most interesting corollary among those we derive, we assert that, for a class $\mathscr{C}$ of monoids or groups possessing a nontrivial member, $\mathrm{G}(\mathscr{C})$ also lacks a finite quasi-equational basis (Corollary 3 ).

We present results from the literature that are related to our work.

O.M. Gornostaev proved that $\mathrm{QG}\left(\mathbb{Z}_{2},+\right)$ and $\mathrm{QG}\left(\mathbb{Z}_{2}, \vee\right)$ are not finitely axiomatizable [10], see also [8, Sect. 6.2]. This sharply contrasts with the facts that all two element algebras have finite equational $[2,13]$ and quasi-equational $[7,16,17]$ bases. Our motivation was to check whether Gornostaev's result is just a curious exception or there is a deeper reason for it.

The existence of definable graphs of semigroups may prevent the existence of finite quasi-equational basis for algebras. D. Casperson and J. Hyndman proved recently that if $\mathbf{A}$ is a finite unary algebra possessing a definable graph of a nontrivial group multiplication as a primitive positive formula, then $\mathrm{Q}(\mathbf{A})$ is not finitely axiomatizable [6].

Surprisingly, almost all finite semigroups generate non finitely axiomatizable quasivarieties [11] in the following sense: If $r(n)$ is the fraction of all semigroups on 
the set $\{0,1, \ldots, n-1\}$ which generate finitely axiomatizable quasivarieties, then $\lim _{n \rightarrow \infty} r(n)=0$. It stays in contrast with the situation for general algebras as almost all finite algebras, in a given finite language, have finite quasi-equational bases. This result was obtained in [11] by M. Jackson and M. Volkov by showing that all finite proper 3-nilpotent semigroups do not have finite quasi-equational bases and applying the result due to D.J. Kleitman, B.R. Rothschild and J.H. Spencer [12] that almost all finite semigroups are proper 3-nilpotent. We refer to [11] for more details. The reader may find there also a discussion about older results concerning finite axiomatizability of quasivarieties of semigroups.

Now we switch our attention to relational structures. There are only five finitely axiomatizable quasivarieties generated by a finite family of finite loopless graphs (relational structures with one binary relation which is symmetric and irreflexive): $\mathrm{Q}(\emptyset), \mathrm{Q}(\bullet), \mathrm{Q}(\bullet \bullet), \mathrm{Q}(\bullet \bullet)$ ) and $\mathrm{Q}(\bullet \bullet \bullet)$. As indicated by X. Caicedo [5, Corollary 3], this fact easily follows from J. Nešetřil and A. Pultr theorem [15, Theorem 3.2]: There are exactly five pseudoquasivarieties (classes of finite structures that are closed under taking substructures and finite direct products) finitely generated by loopless graphs that may be defined by a finite family of finite forbidden subgraphs. Indeed, by Characterization from the next section, a quasivariety generated by graphs is finitely axiomatizable iff it may be defined by a finite family of finite forbidden subgraphs, which in turn is equivalent to the fact that finite members of it form a pseudoquasivariety defined by a finite family of finite forbidden subgraphs.

Other examples of non finitely axiomatizable quasivarieties of relational structures come from studying constraint satisfaction problem. For a finite relational structure $\mathbf{M}$ in a finite language let $\mathbf{A}(\mathbf{M})$ be the class of relational structures that admit a homomorphism into $\mathbf{M}$ (i.e. the antivariety generated by $\mathbf{M}$, see [8]). The class of finite members of $A(\mathbf{M})$ is traditionally denoted by $\operatorname{CSP}(\mathbf{M})$. The class $A(\mathbf{M}) \cup\{\mathbf{L o o p}\}$, where Loop is the one element relational structure in the language of $\mathbf{M}$ with all relations full, is a (finitely generated, see [8, Theorem 3.1.16]) quasivariety. Moreover, the finite axiomatizability of $\mathbf{A}(\mathbf{M})$ is equivalent to the finite axiomatizability of $\mathbf{A}(\mathbf{M}) \cup\{$ Loop $\}$. A relational structure $\mathbf{M}$ has finite duality if there is a finite number of finite relational structures $\mathbf{O}_{1}, \ldots, \mathbf{O}_{n}$, called obstructions, such that each finite relational structure $\mathbf{N}$ admits a homomorphism into $\mathbf{M}$ iff $\mathbf{O}_{1}, \ldots, \mathbf{O}_{n}$ do not admit a homomorphism into $\mathbf{N}$. We will sketch the proof that $\mathbf{M}$ has finite duality if and only if $\mathbf{A}(\mathbf{M})$ is finitely axiomatizable. Most relational structures do not have finite duality [4], and hence we may infer that most quasivarieties of the form $A(\mathbf{M}) \cup\{$ Loop $\}$ are not finitely axiomatizable.

So assume that $\mathbf{A}(\mathbf{M})$ is finitely axiomatizable. Because $A(\mathbf{M})$ is universal, by Characterization in the next section, $\mathrm{A}(\mathbf{M})$ is defined by a finite family $\mathscr{F}$ of finite forbidden substructures. The family $\mathscr{F}$ may be also used as a set of obstructions witnessing finite duality for $\mathbf{M}$. Indeed: if $\mathbf{N} \notin A(\mathbf{M})$, then there is a homomorphism, an embedding, of some $\mathbf{O} \in \mathscr{F}$ into $\mathbf{N}$; if some $\mathbf{O}$ from $\mathscr{F}$ admits a homomorphism into $\mathbf{N}$, then $\mathbf{N} \notin A(\mathbf{M})$, for otherwise $\mathbf{O} \in \mathbf{A}(\mathbf{M})$, which would give us a contradiction. For the converse assume that $\mathbf{M}$ has finite duality witnessed by obstructions $\mathbf{O}_{1}, \ldots, \mathbf{O}_{n}$. Observe that there is a universal sentence $\varphi$ such that $\mathbf{N}$ satisfies it iff none of $\mathbf{O}_{1}, \ldots, \mathbf{O}_{n}$ admits a homomorphism into $\mathbf{N}$. Because $\mathrm{A}(\mathbf{M})$ is universal, $\mathbf{N}$ belongs to $A(\mathbf{M})$ iff all finite substructures of $\mathbf{N}$ belong to $\operatorname{CSP}(\mathbf{M})$ iff all finite 
substructures of $\mathbf{N}$ satisfy $\varphi$ iff $\mathbf{N}$ satisfies $\varphi$. It is worth mentioning that, as proved by A. Atserias [1] and B. Rossman [18], in fact having finite duality by $\mathbf{M}$ is equivalent to the finite axiomatizability of $\operatorname{CSP}(\mathbf{M})$ relative to the class of all finite relational structures in the language of M. But this is a sophisticated result, contrary to the equivalence described above.

Recalled examples together with ours may suggest that quasivarieties of relational structures "have a tendency" to be a non finitely axiomatizable. We do not know to what extent this supposition is true.

\section{Proof}

The proof is based on the following observation which goes back to R.L. Vaught [19].

Observation Assume that $\mathscr{K}$ is a class of relational structures axiomatized by a finite set $\Phi$ of universal sentences. Let $n$ be the maximum number of variables occurring in the sentences from $\Phi$. Then for each relational structure $\mathbf{M}$

$\mathbf{M}$ belongs to $\mathscr{K}$ if all its at most $n$-element substructures do.

The converse is true in the following sense: Let $\mathscr{K}$ be a class of relational structures in a finite language. If there exists a natural number $n$ such that $\left(\boldsymbol{\mho}^{n}\right)$ holds for all relational structures, then $\mathscr{K}$ is finitely axiomatizable. Thus we obtain the following characterization.

Characterization A universal class in a finite relational language is finitely axiomatizable if and only if it may be defined by a finite family of finite forbidden substructures.

Let $\mathscr{C}$ be a class of semigroups possessing a nontrivial member $\mathbf{S}$ with a neutral element. By Observation, in order to prove that $\mathrm{QG}(\mathscr{C})$ is not finitely axiomatizable it is enough to find for each positive integer $n$ a relational structure $\mathbf{M}$ such that

- M does not belong to the quasivariety generated by all graphs of semigroups;

- all at most $n$-element substructures of $\mathbf{M}$ are substructures of powers of $\mathrm{G}(\mathbf{S})$.

The first condition yields $\mathbf{M} \notin \mathrm{QG}(\mathscr{C})$, while the second condition guarantees that all at most $n$-element substructures of $\mathbf{M}$ belong to $Q G(\mathscr{C})$. Hence $\mathbf{M}$ does not fulfill the condition $\left(\Omega^{n}\right)$ for $\mathscr{K}=\mathrm{QG}(\mathscr{C})$.

We start constructing $\mathbf{M}$ by defining its carrier set.

$$
M=\left\{a_{0}, a_{1}, a_{0}^{\prime}, a_{1}^{\prime}, b, c_{0}, \ldots, c_{n}, d_{0}, \ldots, d_{n}, d_{0}^{\prime}, \ldots, d_{n}^{\prime}, e\right\} .
$$

For $k \leqslant n$ let

$$
M_{k}=M-\left\{c_{k}\right\},
$$

and $\varepsilon_{k}: M_{k} \rightarrow \mathbb{Z}_{2}^{n+6}$ be the 1-1 mapping given in Table 1 . 
Table 1 The mapping $\varepsilon_{k}$.

Elements of $\mathbb{Z}_{2}^{n+6}$ are represented as words over $\mathbb{Z}_{2}$. For the sake of clarity we divided these words into 3 segments of length $4, n+1$ and 1 respectively. In the second segment $(k-1)$ th, $k$ th and $(k+1)$ th digits, counting from 0 , are placed between dots

\begin{tabular}{|c|c|c|c|c|}
\hline \multirow{2}{*}{$\begin{array}{l}\text { Elements of } M_{k} \\
a_{0}\end{array}$} & & \multicolumn{3}{|c|}{ Elements of $\mathbb{Z}_{2}^{n+6}$} \\
\hline & & 1100 & $000 \cdots 000 \cdots 000$ & 0 \\
\hline$a_{1}$ & & 0011 & $000 \cdots 000 \cdots 000$ & 0 \\
\hline$a_{0}^{\prime}$ & $\mapsto$ & 1010 & $000 \cdots 000 \cdots 000$ & 0 \\
\hline$a_{1}^{\prime}$ & & 0101 & $000 \cdots 000 \cdots 000$ & 0 \\
\hline$b$ & $\mapsto$ & 1111 & $000 \cdots 000 \cdots 000$ & 0 \\
\hline$c_{0}$ & & 0000 & $100 \cdots 000 \cdots 000$ & 0 \\
\hline$c_{1}$ & & 0000 & $010 \cdots 000 \cdots 000$ & 0 \\
\hline$\cdots$ & & & $\ldots \ldots \ldots \ldots \ldots$ & \\
\hline$c_{k-1}$ & $\mapsto$ & 0000 & $000 \cdots 100 \cdots 000$ & 0 \\
\hline$c_{k+1}$ & & 0000 & $000 \cdots 001 \cdots 000$ & 0 \\
\hline$\cdots$ & & & $\ldots \ldots \ldots \ldots \ldots$ & \\
\hline$c_{n}$ & & 0000 & $000 \cdots 000 \cdots 001$ & 0 \\
\hline$d_{0}$ & & 0011 & $100 \cdots 000 \cdots 000$ & 0 \\
\hline$d_{1}$ & & 0011 & $110 \cdots 000 \cdots 000$ & 0 \\
\hline$\cdots$ & & & $\ldots \ldots \ldots \ldots$ & \\
\hline$d_{k-1}$ & $\mapsto$ & 0011 & $111 \cdots 100 \cdots 000$ & 0 \\
\hline$d_{k}$ & & 0011 & $111 \cdots 110 \cdots 000$ & 1 \\
\hline$d_{k+1}$ & & 0011 & $111 \cdots 111 \cdots 000$ & 1 \\
\hline$\cdots$ & & & $\ldots \ldots \ldots \ldots \ldots$ & \\
\hline$d_{n}$ & & 0011 & $111 \cdots 111 \cdots 111$ & 1 \\
\hline$\overline{d_{0}^{\prime}}$ & & 0101 & $100 \cdots 000 \cdots 000$ & 0 \\
\hline$d_{1}^{\prime}$ & & 0101 & $110 \cdots 000 \cdots 000$ & 0 \\
\hline$\cdots$ & & & $\ldots \ldots \ldots \ldots \ldots$ & \\
\hline$d_{k-1}^{\prime}$ & $\mapsto$ & 0101 & $111 \cdots 100 \cdots 000$ & 0 \\
\hline$d_{k}^{\prime}$ & & 0101 & $111 \cdots 110 \cdots 000$ & 0 \\
\hline$d_{k+1}^{\prime}$ & & 0101 & $111 \cdots 111 \cdots 000$ & 0 \\
\hline$\cdots$ & & & $\ldots \ldots \ldots \ldots \ldots$ & \\
\hline$d_{n}^{\prime}$ & & 0101 & $111 \cdots 111 \cdots 111$ & 0 \\
\hline $\bar{e}$ & $\mapsto$ & 1111 & $111 \cdots 111 \cdots 111$ & 1 \\
\hline
\end{tabular}

Further we need to consider three cases:

- $\left(\mathbb{Z}_{l},+\right)$ for some $l>2$, or $(\mathbb{N},+)$ is isomorphic to a subsemigroup of $\mathbf{S}$. Then define $\mathbf{U}^{>}=\left(\mathbb{Z}_{l}^{n+6},+\right)$ or $\mathbf{U}^{>}=\left(\mathbb{N}^{n+6},+\right)$ respectively;

- $\left(\mathbb{Z}_{2},+\right)$ is isomorphic to a subsemigroup of $\mathbf{S}$. Then define $\mathbf{U}^{2}=\left(\mathbb{Z}_{2}^{n+6},+\right)$;

- $\left(\mathbb{Z}_{2}, \vee\right)$ is isomorphic to a subsemigroup of $\mathbf{S}$. Then define $\mathbf{U}^{\vee}=\left(\mathbb{Z}_{2}^{n+6}, \vee\right)$.

For $\diamond \in\{>, 2, \vee\}, k \leqslant n$, define

$$
\begin{aligned}
R_{k}^{\diamond} & =\left\{(x, y, z) \in M_{k} \mid \mathbf{U}^{\diamond}=\varepsilon_{k}(x) \cdot \varepsilon_{k}(y)=\varepsilon_{k}(z)\right\} \\
& =\left\{(x, y, z) \in M_{k}\left|\mathrm{G}\left(\mathbf{U}^{\diamond}\right)\right|=R\left(\varepsilon_{k}(x), \varepsilon_{k}(y), \varepsilon_{k}(z)\right)\right\}
\end{aligned}
$$

and

$$
\mathbf{M}_{k}^{\diamond}=\left(M_{k}, R_{k}^{\diamond}\right)
$$


Then $\varepsilon_{k}$ is an isomorphism of $\mathbf{M}_{k}^{\diamond}$ onto a substructure of $\mathrm{G}\left(\mathbf{U}^{\diamond}\right)$. The case $\diamond=>$ includes two possibilities $\mathbf{U}^{>}=\left(\mathbb{Z}_{l}^{n+6},+\right)$ and $\mathbf{U}^{>}=\left(\mathbb{N}^{n+6},+\right)$ but in either situation the relations $R_{k}^{>}$are the same. Finally, we take

$$
R^{\diamond}=\bigcup_{k=0}^{n} R_{k}^{\diamond}
$$

and

$$
\mathbf{M}^{\diamond}=\left(M, R^{\diamond}\right)
$$

Claim $\mathbf{M}^{\diamond}$ does not belong to the quasivariety generated by all graphs of semigroups.

Proof In all semigroups the quasi-identity

$$
\left(\forall \bar{x}, \bar{x}^{\prime}, \bar{z}\right)\left[x_{0} x_{1} \approx x_{0}^{\prime} x_{1}^{\prime} \rightarrow x_{0}\left(\cdots\left(x_{1} z_{0}\right) z_{1} \cdots z_{n}\right) \approx x_{0}^{\prime}\left(\cdots\left(x_{1}^{\prime} z_{0}\right) z_{1} \cdots z_{n}\right)\right]
$$

is valid. From this we infer that the quasi-identity

$$
\begin{aligned}
& \left(\forall \bar{x}, \bar{x}^{\prime}, y, \bar{z}, \bar{u}, \bar{u}^{\prime}, v\right)\left[R\left(x_{0}, x_{1}, y\right) \wedge R\left(x_{0}^{\prime}, x_{1}^{\prime}, y\right)\right. \\
& \quad \wedge R\left(x_{1}, z_{0}, u_{0}\right) \wedge R\left(u_{0}, z_{1}, u_{1}\right) \wedge \cdots \wedge R\left(u_{n-1}, z_{n}, u_{n}\right) \wedge R\left(x_{0}, u_{n}, v\right) \\
& \left.\quad \wedge R\left(x_{1}^{\prime}, z_{0}, u_{0}^{\prime}\right) \wedge R\left(u_{0}^{\prime}, z_{1}, u_{1}^{\prime}\right) \wedge \cdots \wedge R\left(u_{n-1}^{\prime}, z_{n}, u_{n}^{\prime}\right) \rightarrow R\left(x_{0}^{\prime}, u_{n}^{\prime}, v\right)\right]
\end{aligned}
$$

holds in each graph $(W, R)$ of a semigroup W. Indeed, if the premises of $(\boldsymbol{Z})$ are satisfied under a valuation $\rho$ : Variable $\rightarrow W$, then in $\mathbf{W}$

$$
\begin{aligned}
\rho(y) & =\rho\left(x_{0}\right) \rho\left(x_{1}\right)=\rho\left(x_{0}^{\prime}\right) \rho\left(x_{1}^{\prime}\right), \\
\rho\left(u_{n}\right) & =\left(\cdots\left(\rho\left(x_{1}\right) \rho\left(z_{0}\right)\right) \rho\left(z_{1}\right) \cdots\right) \rho\left(z_{n}\right), \\
\rho\left(u_{n}^{\prime}\right) & =\left(\cdots\left(\rho\left(x_{1}^{\prime}\right) \rho\left(z_{0}\right)\right) \rho\left(z_{1}\right) \cdots\right) \rho\left(z_{n}\right)
\end{aligned}
$$

and by (ð)

$$
\begin{aligned}
\rho(v)=\rho\left(x_{0}\right) \rho\left(u_{n}\right) & =\rho\left(x_{0}\right)\left(\left(\cdots\left(\rho\left(x_{1}\right) \rho\left(z_{0}\right)\right) \rho\left(z_{1}\right) \cdots\right) \rho\left(z_{n}\right)\right) \\
& =\rho\left(x_{0}^{\prime}\right)\left(\left(\cdots\left(\rho\left(x_{1}^{\prime}\right) \rho\left(z_{0}\right)\right) \rho\left(z_{1}\right) \cdots\right) \rho\left(z_{n}\right)\right)=\rho\left(x_{0}^{\prime}\right) \rho\left(u_{n}^{\prime}\right) .
\end{aligned}
$$

Now consider the valuation $\delta$ : Variable $\rightarrow M$;

$$
\begin{aligned}
& x_{0} \mapsto a_{0}, \quad x_{1} \mapsto a_{1}, \quad x_{0}^{\prime} \mapsto a_{0}^{\prime}, \quad x_{1}^{\prime} \mapsto a_{1}^{\prime}, \\
& y \mapsto b, \quad z_{k} \mapsto c_{k}, \quad u_{k} \mapsto d_{k}, \quad u_{k}^{\prime} \mapsto d_{k}^{\prime}, \quad v \mapsto e
\end{aligned}
$$

for $k \leqslant n$. The straightforward verification shows that

$$
\begin{aligned}
& \left(a_{0}, a_{1}, b\right),\left(a_{0}^{\prime}, a_{1}^{\prime}, b\right) \in R_{0}^{\diamond}, \\
& \left(a_{1}, c_{0}, d_{0}\right) \in R_{1}^{\diamond}, \quad\left(d_{0}, c_{1}, d_{1}\right), \ldots,\left(d_{n-1}, c_{n}, d_{n}\right),\left(a_{0}, d_{n}, e\right) \in R_{0}^{\diamond}, \\
& \left(a_{1}^{\prime}, c_{0}, d_{0}^{\prime}\right) \in R_{1}^{\diamond}, \quad\left(d_{0}^{\prime}, c_{1}, d_{1}^{\prime}\right), \ldots,\left(d_{n-1}^{\prime}, c_{n}, d_{n}^{\prime}\right) \in R_{0}^{\diamond} .
\end{aligned}
$$


Thus, under the valuation $\delta$, the premises in the quasi-identity ( $\zeta$ ) are satisfied. However $\left(a_{0}^{\prime}, d_{n}^{\prime}, e\right)$ does not belong to any $R_{k}^{\diamond}$, and hence ( $\zeta$ ) fails in $\mathbf{M}^{\diamond}$.

Lemma $2 R^{\diamond} \cap M_{k}^{3}=R_{k}^{\diamond}$.

Proof Let $x, y, z \in M_{k}$ and $\varepsilon_{l}(x) \cdot \varepsilon_{l}(y)=\varepsilon_{l}(z)$, i.e. $(x, y, z) \in R_{l}^{\diamond}$, for some $l \leqslant n$. Our aim is to prove that $(x, y, z) \in R_{k}^{\diamond}$.

Suppose first that at least two of $x, y, z$ are equal. Then $\diamond=\vee$. Indeed, if $x=y$, then 2 would have to occur in $\varepsilon_{l}(z)$ when $\diamond=>$, or $\varepsilon_{l}(z)$ would be the tuple of zeros when $\diamond=2$. If $x=z$ or $y=z$, one of $\varepsilon_{l}(x), \varepsilon_{l}(y)$ would be the tuple of zeros in both cases. Thus, say, $\varepsilon_{l}(x) \leqslant \varepsilon_{l}(y)=\varepsilon_{l}(z)$ in the order of the semilattice $\mathbf{U}^{\vee}$. For any $u$, it is the case that $\varepsilon_{k}(u)$ and $\varepsilon_{l}(u)$ are the same except possibly for the last digit. This is why the uncertainty may occur only when $\varepsilon_{k}(x)$ ends with 1 . This means $x=e$, which forces $y=z=e$, or $x=d_{i}$ for some $i \geqslant k$. In the latter case, by the definition of $\varepsilon_{k}$, we must have $y=z \in\left\{d_{i}, d_{i+1}, \ldots, d_{n}, e\right\}$, and hence $\varepsilon_{k}(y)$ ends with 1 , too. Therefore, in both cases, $(x, y, z) \in R_{k}^{\vee}$.

Now suppose that $x, y, z$ are distinct. We may assume that $\left.\varepsilon_{l}\right|_{\{x, y, z\}} \neq\left.\varepsilon_{k}\right|_{\{x, y, z\}}$, which implies that $d_{i} \in\{x, y, z\}$ for some $i<n$. Then either $\{x, y, z\}=\left\{a_{1}, c_{0}, d_{0}\right\}$ and $k, l>0$, or $\{x, y, z\}=\left\{d_{j}, c_{j+1}, d_{j+1}\right\}$, for some $j$, and $k, l \neq j+1$. In both cases we again conclude that $\varepsilon_{k}(x) \cdot \varepsilon_{k}(y)=\varepsilon_{k}(z)$.

Claim Each substructure $\mathbf{N}$ of $\mathbf{M}^{\diamond}$ having at most $n$ elements is isomorphic to a substructure of a power of $\mathrm{G}(\mathbf{S})$.

Proof By Lemma 2, $\mathbf{N}$ is a substructure of some $\mathbf{M}_{k}^{\diamond}$. We defined $\mathbf{M}_{k}^{\diamond}$ in such a way that it is isomorphic to a substructure of $\mathrm{G}\left(\mathbf{U}^{\diamond}\right) \leqslant \mathrm{G}\left(\mathbf{S}^{n+6}\right)=\mathrm{G}(\mathbf{S})^{n+6}$.

\section{Related results}

We start by demonstrating how small modifications in the proof of Theorem 1 give the following result.

Corollary 3 Let $\mathscr{C}$ be a class of monoids or groups possessing a nontrivial member. Then $\mathrm{QG}(\mathscr{C})$ is not finitely axiomatizable.

Proof In the monoid case let $\mathbf{U}_{m}^{\diamond}$ be the monoid with the semigroup reduct $\mathbf{U}^{\diamond}$. As previously, we define $\mathbf{M}_{m, k}^{\diamond}=\left(M_{k}, R_{\cdot, k}^{\diamond}, R_{1, k}\right), k \leqslant n$, in such a way that $\varepsilon_{k}$ is an isomorphism of $\mathbf{M}_{m, k}^{\diamond}$ onto a substructure of $\mathrm{G}\left(\mathbf{U}_{m}^{\diamond}\right)$. Note that $R_{1, k}^{\diamond}=\emptyset$, and thus the reasoning from the proof of Theorem 1 remains correct here.

In the group case a slightly larger modification is needed. We define $\mathbf{U}_{g}^{>}$to be the group $\left(\mathbb{Z}_{l}^{n+6},+,-, 0\right), l>2$, or $\left(\mathbb{Z}^{n+6},+,-, 0\right)$, and $\mathbf{U}_{g}^{2}$ to be the group $\left(\mathbb{Z}_{2}^{n+6},+,-, 0\right)$. Then, in analogically constructed $\mathbf{M}_{g}^{\diamond}=\left(M, R ., R_{()^{-1}}^{\diamond}, R_{1}\right)$, we have $R_{1}^{\diamond}=\emptyset, R_{()^{-1}}^{>}=\emptyset$ and $R_{()^{-1}}^{2}=\{(a, a) \mid a \in M\}$. 
Now we consider fragments of quasi-equational theories.

Recall that by an identity we mean a sentence of the form $(\forall \bar{x}) \varphi(\bar{x})$, where $\varphi$ is an atomic formula. A variety is a class defined by identities. The following simple statement shows that the problem of finite axiomatizability of varieties of relational structures is not interesting.

Proposition 4 Let $\mathscr{K}$ be a variety in a finite relational language. Then $\mathscr{K}$ is finitely axiomatizable.

Proof It follows from the fact that there is, up to renaming of variables, only finite number of identities in a finite relational language.

Here is a more peculiar result. For a class $\mathscr{K}$ let $\mathrm{Q}_{\not}(\mathscr{K})$ be the class defined by all quasi-identities true in $\mathscr{K}$ in which the equality symbol $\approx$ does not occur. Quasiidentities without the equality symbol form a fragment of first order logic particularly important in abstract algebraic logic [3].

Corollary 5 Let $\mathscr{C}$ be a class of monoids or groups possessing a nontrivial member, or of semigroups possessing a nontrivial member with a neutral element. Then $\mathrm{Q}_{\approx} \mathrm{G}(\mathscr{C})$ is not finitely axiomatizable.

Proof The quasi-identity ( $\mathbf{Z}$ ) was chosen in such a way that it also serves here. The proof of Theorem 1 may be used without any change.

After obtaining Theorem 1 the author learned that Gornostaev proved a similar fact. We formulate it as follows. By a reduction of a relational structure $(M, R)$ we mean any relational structure $\left(M, R^{\prime}\right)$, where $R^{\prime} \subseteq R$. Let $\mathrm{R}(\mathscr{K})$ stand for the class of all reductions of relational structures from a class $\mathscr{K}$. We will prove in Appendix that $\mathrm{RQ}(\mathscr{K})$ is the quasivariety defined by the quasi-identities true in $\mathscr{K}$ whose conclusions are of the form $x \approx y$.

Theorem 6 (Gornostaev [9]) Let $\mathscr{C}$ be a class of semigroups possessing a nontrivial member with a neutral element. Then $\mathrm{RQG}(\mathscr{C})$ is not finitely axiomatizable.

We will provide a sketch of the proof for the publication [9] is scarcely available.

Proof The main idea is again the same, but details are simpler. We cannot use the quasi-identity ( $\zeta$ ) for in the conclusion of it the symbol $R$ was used. But we may use the following one

$$
\begin{aligned}
& \left(\forall \bar{x}, \bar{x}^{\prime}, y, \bar{z}, \bar{u}, \bar{u}^{\prime}, v, v^{\prime}\right)\left[R\left(x_{0}, x_{1}, y\right) \wedge R\left(x_{0}^{\prime}, x_{1}^{\prime}, y\right)\right. \\
& \quad \wedge R\left(x_{1}, z_{0}, u_{0}\right) \wedge R\left(u_{0}, z_{1}, u_{1}\right) \wedge \cdots \wedge R\left(u_{n-1}, z_{n}, u_{n}\right) \wedge R\left(x_{0}, u_{n}, v\right) \\
& \quad \wedge R\left(x_{1}^{\prime}, z_{0}, u_{0}^{\prime}\right) \wedge R\left(u_{0}^{\prime}, z_{1}, u_{1}^{\prime}\right) \wedge \cdots \wedge R\left(u_{n-1}^{\prime}, z_{n}, u_{n}^{\prime}\right) \wedge R\left(x_{0}^{\prime}, u_{n}^{\prime}, v^{\prime}\right) \\
& \left.\quad \rightarrow v \approx v^{\prime}\right] .
\end{aligned}
$$


Instead of three $\mathbf{M}^{\diamond}$ it is enough to define one relational structure $\mathbf{M}^{\prime}=\left(M^{\prime}, R^{\prime}\right)$, where $M^{\prime}=M \cup\left\{e^{\prime}\right\}$ and

$$
\begin{aligned}
R^{\prime}= & \left(a_{0}, a_{1}, b\right),\left(a_{0}^{\prime}, a_{1}^{\prime}, b\right), \\
& \left(a_{1}, c_{0}, d_{0}\right),\left(d_{0}, c_{1}, d_{1}\right), \ldots\left(d_{n-1}, c_{n}, d_{n}\right), \\
& \left(a_{1}^{\prime}, c_{0}, d_{0}^{\prime}\right),\left(d_{0}^{\prime}, c_{1}, d_{1}^{\prime}\right), \ldots\left(d_{n-1}^{\prime}, c_{n}, d_{n}^{\prime}\right), \\
& \left.\left(a_{0}, d_{n}, e\right),\left(a_{0}^{\prime}, d_{n}, e^{\prime}\right)\right\} .
\end{aligned}
$$

Then $\mathbf{M}^{\prime}=\left(M^{\prime}, R^{\prime}\right)$ does not model ( $\left.\boldsymbol{\zeta}^{\prime}\right)$. For $k<n$ let $M_{k}^{\prime}=M^{\prime}-\left\{c_{k}\right\}, \mathbf{M}_{k}^{\prime}$ be the substructure of $\mathbf{M}^{\prime}$ with the carrier set $M_{k}^{\prime}$, and $\varepsilon_{k}^{\prime}$ be the extension of $\varepsilon_{k}$ mapping $e^{\prime}$ onto the tuple of $n+5$ ones and one zero at the end. Then $\varepsilon_{k}^{\prime}: \mathbf{M}_{k}^{\prime} \rightarrow \mathbf{G}\left(\mathbf{U}^{\diamond}\right)$ is a 1-1 homomorphism. The original proof is similar.

It was not indicated by Gornostaev, but this result clearly holds for classes of monoids or groups containing a nontrivial member. Just, in the proof above, define the relations corresponding to graphs of operations 1 and ()$^{-1}$ to be the empty set.

Acknowledgements The main result of this paper, i.e. Theorem 1, was obtained during the author's visit in University of Puerto Rico at Mayagüez. We would like to express our deep gratitude to Wiesław Dziobiak for the inspiration and kind hospitality. We are grateful to the anonymous referee for her or his suggestions that helped improving the paper significantly. In particular, Proposition 7 was observed by her or him. We also would like to thank Marina V. Semenova for providing us the articles [9, 10].

Open Access This article is distributed under the terms of the Creative Commons Attribution Noncommercial License which permits any noncommercial use, distribution, and reproduction in any medium, provided the original author(s) and source are credited.

\section{Appendix}

Here we take a closer look at the reduction class operator. First we extend the definition to models in an arbitrary language. Let $\mathbf{M}=(M, R, O)$ be a model, where $R=\left\{R_{\gamma} \mid \gamma \in \Gamma\right\}$ is an indexed set of relations, and $O$ is a set of operations. A reduction of $\mathbf{M}$ is any model of the form $\left(M, R^{\prime}, O\right)$, where $R^{\prime}=\left\{R_{\gamma}^{\prime} \mid \gamma \in \Gamma\right\}$ and $R_{\gamma}^{\prime} \subseteq R_{\gamma}$ for all $\gamma \in \Gamma$. As previously, $\mathrm{R}(\mathscr{K})$ stands for the class of all reductions of models from $\mathscr{K}$.

Proposition 7 For an arbitrary quasivariety $\mathscr{Q}$ the class $\mathrm{R}(\mathscr{Q})$ is the quasivariety defined by the quasi-identities true in $\mathscr{Q}$ whose conclusions are equalities of terms.

Proof We divide the set $\Lambda$ of all quasi-identities satisfied in $\mathscr{Q}$ into two sets: $\Lambda_{R}$ of those with conclusions containing a relational symbol different from $\approx$, and $\Lambda_{O}$ of those with conclusions being equalities of terms. That $\Lambda_{O}$ holds in $\mathrm{R}(\mathscr{Q})$ is fairly straightforward.

Take an arbitrary model $M=\left(M, R^{\prime}, O\right)$ satisfying $\Lambda_{O}$. We define relations $R_{\gamma}$, $\gamma \in \Gamma$, on the set $M$ in such a way that each $R_{\gamma}$ contains $R_{\gamma}^{\prime}$ and $(M, R, O) \in \mathscr{Q}$. 
Precisely, let $R_{\gamma}$ be the set of all tuples $\bar{a}$ of elements in $M$ for which there exist a quasi-identity

$$
(\forall \bar{y})\left[\psi_{1}(\bar{y}) \wedge \cdots \wedge \psi_{m}(\bar{y}) \rightarrow \psi(\bar{y})\right] \in \Lambda_{R}
$$

and a tuple $\bar{b}$ such that $\left(M, R^{\prime}, O\right) \models \psi_{i}(\bar{b})$ for $i \leqslant m$, and $\psi(\bar{b})=R_{\gamma}(\bar{a})$. The fact that $(\forall \bar{y})\left[R_{\gamma}(\bar{y}) \rightarrow R_{\gamma}(\bar{y})\right]$ belongs to $\Lambda_{R}$ yields $R_{\gamma}^{\prime} \subseteq R_{\gamma}$. Now take

$$
q=(\forall \bar{x})\left[\varphi_{1}(\bar{x}) \wedge \cdots \wedge \varphi_{n}(\bar{x}) \rightarrow \varphi(\bar{x})\right] \in \Lambda
$$

and a tuple $\bar{a}$ such that $(M, R, O) \models \varphi_{i}(\bar{a})$ for all $i \leqslant n$. Our aim is to verify that $(M, R, O)$ satisfies $\varphi(\bar{a})$. Assume that the formulas $\varphi_{1}, \ldots, \varphi_{k}$ contain relational symbols different from $\approx$, and $\varphi_{k+1}, \ldots, \varphi_{n}$ contain $\approx$. Then for every $i \leqslant k$ there are a quasi-identity

$$
q_{i}=\left(\forall \bar{y}_{i}\right)\left[\psi_{i, 1}\left(\bar{y}_{i}\right) \wedge \cdots \wedge \psi_{i, m_{i}}\left(\bar{y}_{i}\right) \rightarrow \psi_{i}\left(\bar{y}_{i}\right)\right] \in \Lambda_{R}
$$

and a tuple $\bar{b}_{i}$ such that $\psi_{i}\left(\bar{b}_{i}\right)=\varphi_{i}(\bar{a})$ and $\left(M, R^{\prime}, O\right) \models \psi_{i, j}\left(\bar{b}_{i}\right)$ for $j \leqslant m_{i}$. At this stage a bit care about variables is needed. First, we may assume that all valuations $\bar{x} \mapsto \bar{a}, \bar{y}_{i} \rightarrow \bar{b}_{i}, i \leqslant k$, are $1-1$ (identify variables if needed). Then we may rename variables in $\bar{y}_{i}$ in such a way that $\psi_{i}\left(\bar{y}_{i}\right)=\varphi_{i}(\bar{x})$. Finally, assume that if a variable from $\bar{y}_{i}$ does not occur in $\varphi_{i}(\bar{x})$, then it does not occur in $q, q_{1}, \ldots, q_{i-1}, q_{i+1}, \ldots, q_{k}$. Let $q^{\prime}$ be the quasi-identity

$$
\left(\forall \bar{x}, \bar{y}_{1}, \ldots, \bar{y}_{k}\right)\left[\psi_{1,1}\left(\bar{y}_{1}\right) \wedge \cdots \wedge \psi_{k, m_{k}}\left(\bar{y}_{k}\right) \wedge \varphi_{k+1}(\bar{x}) \wedge \cdots \wedge \varphi_{n}(\bar{x}) \rightarrow \varphi(\bar{x})\right] .
$$

Then $q^{\prime} \in \Lambda$ and

$$
\left(M, R^{\prime}, O\right) \models \psi_{1,1}\left(\bar{b}_{1}\right) \wedge \cdots \wedge \psi_{k, m_{k}}\left(\bar{b}_{k}\right) \wedge \varphi_{k+1}(\bar{a}) \wedge \cdots \wedge \varphi_{n}(\bar{a}) .
$$

If $q^{\prime} \in \Lambda_{R}$, then the definition of the relations in $R$ yields $(M, R, O) \models \varphi(\bar{a})$. If $q^{\prime} \in \Lambda_{O}$, then $\left(M, R^{\prime}, O\right) \models q^{\prime}$, and hence $\left(M, R^{\prime}, O\right) \models \varphi(\bar{a})$, which implies $(M, R, O) \models \varphi(\bar{a})$. We conclude that $(M, R, O)$ satisfies $q$.

Corollary 8 A class of models in a given language is definable by quasi-identities whose conclusions are equalities of terms if and only if it is closed under $\mathrm{R}$ and $\mathrm{Q}$ class operators.

Note that there are quasi-identities without $\approx$ in the conclusion that may hold in $\mathrm{RQ}(\mathscr{K})$. Take for instance the tautology $(\forall \bar{x})[R(\bar{x}) \rightarrow R(\bar{x})]$.

\section{References}

1. Atserias, A.: On digraph coloring problems and treewidth duality. Eur. J. Combin. 29(4), 796-820 (2008)

2. Berman, J.: A proof of Lyndon's finite basis theorem. Discrete Math. 29(3), 229-233 (1980)

3. Blok, W.J., Pigozzi, D.: Algebraic semantics for universal Horn logic without equality. In: Universal Algebra and Quasigroup Theory, Jadwisin, 1989. Res. Exp. Math., vol. 19, pp. 1-56. Heldermann, Berlin (1992) 
4. Bulatov, A.A., Krokhin, A., Larose, B.: Dualities for constraint satisfaction problems. In: Creignou, N., et al. (eds.) Complexity of Constraints. An Overview of Current Research Themes. Lecture Notes in Computer Science, vol. 5250, pp. 93-124. Springer, Berlin (2008)

5. Caicedo, X.: Finitely axiomatizable quasivarieties of graphs. Algebra Universalis 34(2), 314-321 (1995)

6. Casperson, D., Hyndman, J.: Primitive positive formulas preventing a finite basis of quasi-equations. Int. J. Algebra Comput. 19(7), 925-935 (2009)

7. Gorbunov, V.A.: Quasiidentities of two-element algebras. Algebra Logic 22(2), 83-88 (1983)

8. Gorbunov, V.A.: Algebraic Theory of Quasivarieties. Consultants Bureau, New York (1998)

9. Gornostaev, O.M.: Quasivarieties of models embeddable into semigroups. Deposited at the All-Union Institute of Scientific and Technical Information, No. 1812-82 (1982) (in Russian)

10. Gornostaev, O.M.: Quasivarieties generated by classes of models. Deposited at the All-Union Institute of Scientific and Technical Information, No. 2206-85 (1985) (in Russian)

11. Jackson, M., Volkov, M.: Relatively inherently nonfinitely $q$-based semigroups. Trans. Am. Math. Soc. 361(4), 2181-2206 (2009)

12. Kleitman, D.J., Rothschild, B.R., Spencer, J.H.: The number of semigroups of order $n$. Proc. Am. Math. Soc. 55(1), 227-232 (1976)

13. Lyndon, R.C.: Identities in two-valued calculi. Trans. Am. Math. Soc. 71(3), 457-465 (1951)

14. Mal'cev, A.I.: Algebraic Systems. Springer, New York (1973)

15. Nešetřil, J., Pultr, A.: On classes of relations and graphs determined by subobjects and factorobjects. Discrete Math. 22(3), 287-300 (1978)

16. Nurakunov, A.M.: Quasivarieties generated by two-element algebras revisited. In: Blatt, H.-P., et al. (eds.) Analytical and Approximate Methods. Proceedings of the International Conference at the Kyrgyz-Russian-Slavic University Bishkek, Kyrgyzstan, September 23-24, 2002. Berichte aus der Mathematik. Shaker Verlag, Aachen (2003)

17. Rautenberg, W.: 2-element matrices. Stud. Log. 40(4), 315-353 (1981)

18. Rossman, B.: Homomorphism preservation theorems. J. ACM 55(3), 1-54 (2008)

19. Vaught, R.L.: Remarks on universal classes of relational systems. Nederl. Akad. Wetensch. Proc. Ser. A 57 (1954). Indag. Math. 16, 589-591 (1954) 